\title{
THE EFFECT OF ATTRACTOR MATERIAL ON PELAGIC FISH CAPTURED USING PAYANG BUGIS IN PASAURAN WATERS, PROVINCE OF BANTEN
}

\author{
Roza Yusfiandayani \\ Lecturer in Faculty of Fisheries and Marine Science, Bogor Agricultural University, Bogor \\ Received November 30-2009; Received in revised form July 26-2010; Accepted August 12-2011
}

\begin{abstract}
Rumpon (fish aggregating device) as an auxiliary gear operated in Pasauran waters, Sunda Strait was longer and more widely used in Indonesia to catch pelagic fish. The characteristic species composition, size, and gonado somato index (GSI) pelagic fish around 3 kinds of rumpon attractor materials, i.e. Cocos nucifera, Nypa fructican, and Areca catechu leaves will determine the effects of attractor materials will affect the sustainability of pelagic fish associated. Identification and composition length, weight and gonado somato index pelagic fish were identified in this research with different attractor material in all seasons. Experimental fishing carried out by using payang Bugis. The result show that the Cocos nucifera leaves are the best attractor material based on the number of fish species and durability in all seasons. Further, the gonado somato index of small pelagic fish were caught around rumpon are gonado somato index I 4\%, gonado somato index II $33 \%$, gonado somato index III 35\%, gonado somato index IV 25\%, and gonado somato index V $3 \%$. Pelagic fish around attractor materials have gonado somato index I-III $72 \%$. This result indicated that the small pelagic fish caught around rumpon are immature fish.
\end{abstract}

\section{INTRODUCTION}

Fish resource is renewable but it has limitation. Based on the condition, utilization of fish resource should be arranged or managed properly to gain optimum utilization by caring the fish resource sustainability.

The existence of pelagic fish as trade commodity from fishery sector in Indonesia is one of the resources that has a large number, both as an export and domestic consumed commodity to fulfill national nutrient need. The pelagic fish can be classified into big pelagic fish and small pelagic fish. The big pelagic fish consist of skipjack tune, big eye tuna, barred spanish mackerel, etc. The small pelagic fish are mackerel, fringescale sardine, rainbow runner, hardtail scad, Indonesian oil sardine, trevally, barred garfish, anchovy, etc.

Fishing gear used generally to capture pelagic fish are drift gillnet, encircling gillnet, seine net, lift net, purse seine, troll line, pole and line, hand line, etc. The fishing gear that uses an auxilliary gear like rumpon has been applied on purse seine and lines that are so helpful in developing captured fish result.

The Sunda Strait waters is one of the straits located in Nusantara waters and it has abundant natural resources that can be used to people's welfare and nutrient developing. To anticipate development the sea as pillar for fishermen and their families, good management enables to implement sustainable development.

The changing condition of waters in every season influences the existence of fish resources, fishermen characteristic, and capture area. The weather around Sunda Strait and Java Ocean is a typical area influenced by wind blowing on west monsoon and east monsoon period. This phenomenon can be seen by the changing condition influenced by characteristic of Java Ocean and Hindian Ocean especially on the peak of the monsoon.

Small scale fishery activity generally captures the fish with limited scope because of limited capital, devices, and skill. These limitations effect to low capture that causes to their low income. To increase the scope of capture area needs much capital where the small scale fishermen can't afford it. One of the alternatives can be done to solve the obstacle is by applying the rumpon technology.

The application of rumpon technology will give certainty on capture area and it is expected to give solution for the problem related to rumpon engineering, material, number, and space, as well as optimum productivity and efficiency of capture.

Globally, both deep sea, and shallow rumpon consist of four main components i.e. float, rope, attractor, and sinker. The attractors commonly used are such as the coconut trees, tyrewall, nets, and 
ropes/strings tied up to the raft have been successful in developing rumpon activity to attract fish. Some traditional fishermen choose natural leaves as the attractors, it is cheaper than using artificial attractors. The natural leaves mainly used as attractors are Cycadaceae like coconut leave (Cocos nucifera), nipa (Nypa fructican), pinang/areca (Areca Catechu) and so on.

Research and theory about fish association around rumpon have been carried out since 1967. Samples and Sproul (1985) stated the theory that attracted fish around rumpon caused by:

1. Rumpon as a shading place for certain fish.

2. Rumpon as feeding ground for certain fish.

3. Rumpon as substratum to put the eggs for certain fish.

4. Rumpon as a shelter from some predators for certain fish.

5. Rumpon as meeting point for certain fish.

The associating fish around attractors from natural leaves caused by difference of attractor material on rumpon has not been known yet. The characteristics of species, size, and maturity of pelagic fish gonado captured around rumpon will determine whether the influence of attractor material used endangers sustainability associating pelagic fish or not. This basic data will be used to arranged rumpon fishery development observed from fish behavior, number of rumpon, and its productivity in a waters connected with sustainability of pelagic fish resource.

\section{Goal}

The goal of this research are:

1. To identify species characteristic and captured pelagic size around rumpon with different attractor materials.

2. To estimate gonado somato index captured pelagic fish around rumpon with different attractor materials.

\section{MATERIALS AND METHODS}

\section{Time and Place}

The research was conducted on December 2001 until September 2002 in Pasauran waters, Sunda Strait (Figure 1). The research session based on season shift 1 (musim peralihan 1) March 2002; east season (musim timur) June 2002; season shift 2 (musim peralihan 2) September 2002; and west season (musim barat) December 2001.

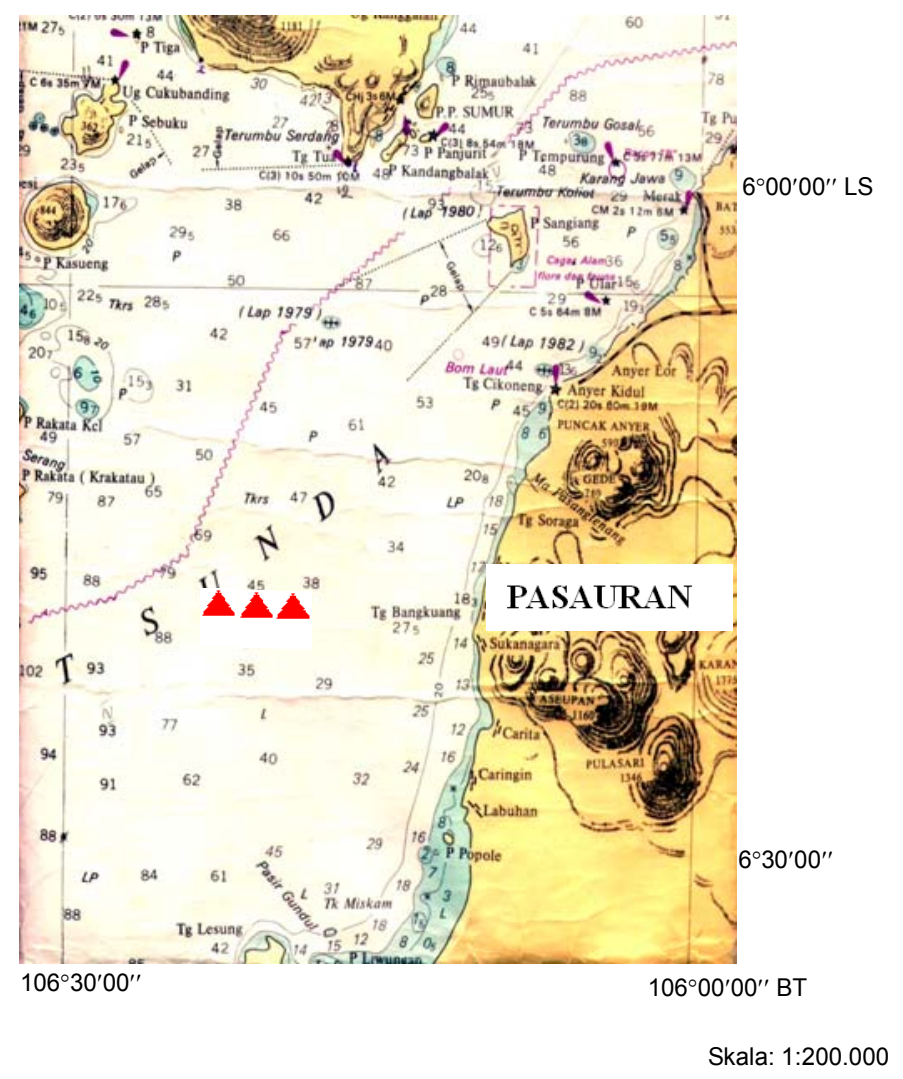

Figure 1. 


\section{Material and Devices}

The materials used in this research are:

1. Rumpon. During the research, the writer used 3 units shallow sea rumpon put in $45 \mathrm{~m}$ depth (Figure 2). Each rumpon had some components 1) 3-6 bamboos used as float. Length and its diameter are 15 and $0.1 \mathrm{~m} ; 2$ ) coconut leaves, areca, and nipa leaves used as natural attractors consist of 25 pieces; 3) attractor and anchor ropes are PE $18 \mathrm{~mm}$ and with length $63 \mathrm{~m}$, binding rope for natural attractor is PE 2-5 mm with its length 30 $50 \mathrm{~cm}$; and 4) sinker consists of two $50 \mathrm{~kg}$ stones. Detailed description of the rumpon can be seen on Table 1.

2. Attractors. Coconut leaves (Cocos nucifera), nipa leaves (Nypa fructican) and areca leaves (Areca catechu). Samples taken from those leaves were 25 pieces which divided into two and binded on the rope.

Table 1. Description of rumpon used on the research

\begin{tabular}{|c|c|c|c|c|c|}
\hline No. & Components & Materials & Size & Amount & Weight \\
\hline \multirow[t]{2}{*}{1.} & Float & & & & \\
\hline & - bamboo raft & bamboo & $\begin{array}{l}\text { length } 15 \mathrm{~m} \\
\text { diameter } 0,1 \mathrm{~m}\end{array}$ & 3-6 stems & - \\
\hline \multirow[t]{4}{*}{2.} & Attractors & & & & \\
\hline & - Natural attractors & Coconut leaves & - & 25 pieces & - \\
\hline & & Nipa leaves & & 25 pieces & \\
\hline & & Areca leaves & & 25 pieces & \\
\hline \multirow{3}{*}{3.} & Attractor rope and anchor & $P F$ & lenath $63 \mathrm{~m}(14 \mathrm{x}$ denth) & $45 \mathrm{~m}$ & - \\
\hline & - - Tope & rᄃ & diameter $18 \mathrm{~mm}$ & 45111 & - \\
\hline & - binder for natural attractors & PE & $\begin{array}{l}\text { length } 30-50 \mathrm{~cm} \\
\text { diameter } 2-5 \mathrm{~mm}\end{array}$ & 1 piece per atraktor & - \\
\hline 4. & $\begin{array}{l}\text { Sinker } \\
\text { - sinker }\end{array}$ & Stone & - & 2 pieces & $100 \mathrm{~kg}$ \\
\hline
\end{tabular}

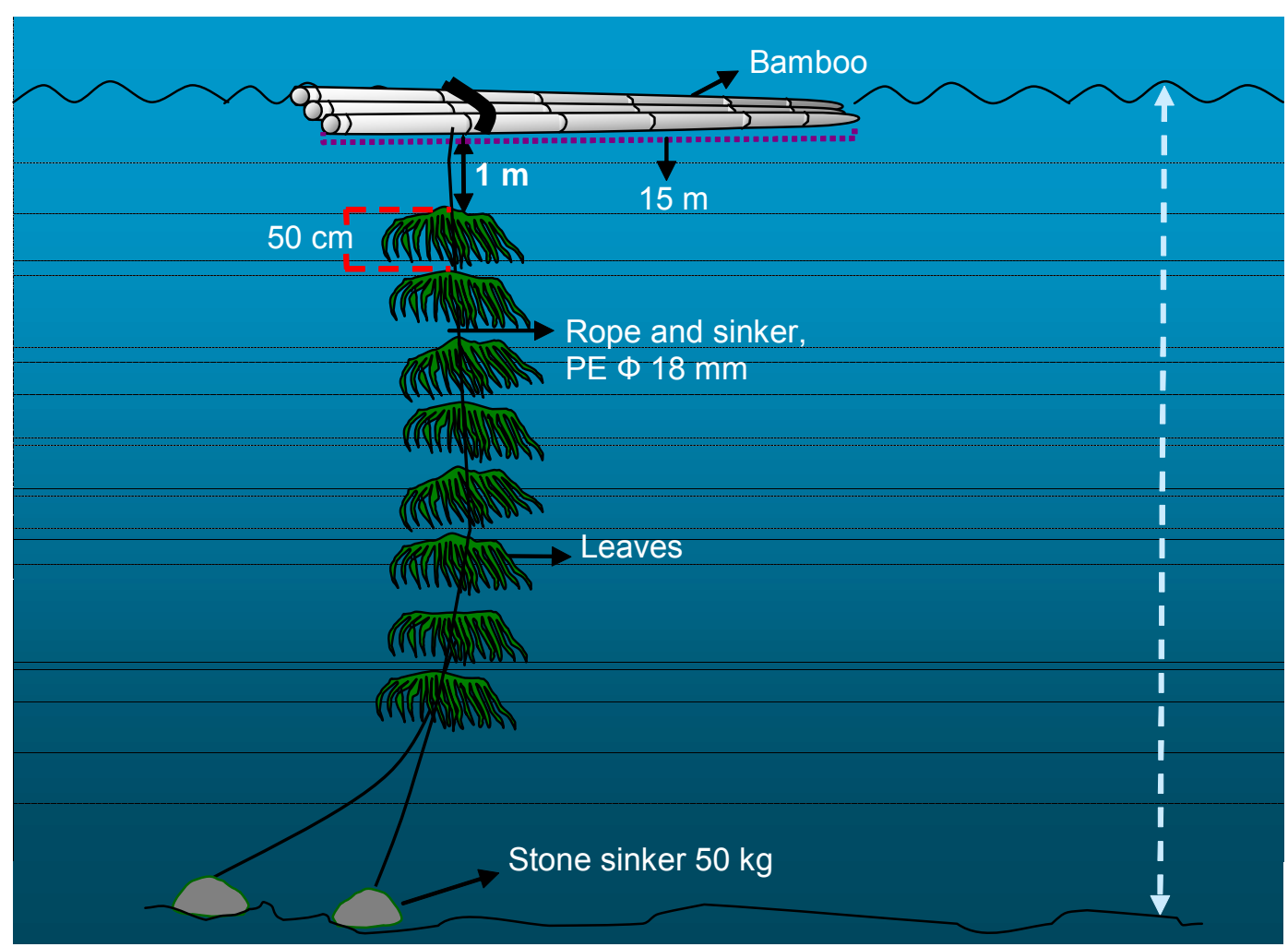

Figure 2. Construction of shallow sea rumpon used in the research. 
Other devices used in this research are:

1. Secchi disc, refraktometer, termometer, global positioning system, sonar, accoustic instruments portable, underwater camera, spektrofotometer ultraviolet, spektrofotometer Beckman, binocular microscope, pipette, object glass, gelas penutup, scissors, sample bottle, tissue, surgery device, preservatives (lugol $40 \%$ dan formalin $4 \%$ ), preservatives formalin acetat acid (faa), xylol, alcohol, parafin, alcian blue, camera, scale, stationary, books: Periphyton and plankton identification" (Yamaji, 1976), and Identification of Fischer and whitehead fish (1974).

2. Capture device. For fish sample observation and evaluation on fish composition around rumpon, the writer used capture device as sampling gear that enables to take some examples represent the population such as payang Bugis.

\section{Research Methodology}

The research was conducted by using experimental fishing and survey methods. Experimental fishing method was conducted by capturing experiment on shallow sea rumpon in Pasauran waters, Sunda Strait. Capture experiment was carried out to each rumpon unit by using payang Bugis device for 5 times. Social survey was conducted by using quisionnaire to 20 fishermen and 2 auction officers. Total amount of payang Bugis fishermen in Pasauran are 50. Primary data derived from interview with some fishermen based on the questionnaire and from active participation in joining the capture operation.

The sample was taken by using experimental fishing method, i.e. capture experiment. Waters condition, range among rumpon, rumpon installation depth, and oceanography condition have influenced in this research. Fish sample was taken by capturing around rumpon with payang Bugis. Total of the rumpon used were 3 units which each unit was conducted to capture fish with payang Bugis. From each capture in every trip, it was measured the total of capture and counted the total of captured pelagic fish. Sub sample taken from dominant species from capturing using payang Bugis with various size from the smallest to the biggest one. According to Nasoetion \& Barizi (1980), before it was analized in detail, the data dissemination of capture result based on attractor materials and different season were tested before to know whether the data spread normally or not. Normality test used was liliefors normality test. After the test had been conducted, if the data didn't spread normally, it would be continued with non parametric test, it was duncan signed rank experiment. The data were processed by using SPSS 10.0 program.

\section{RESULTS AND DISCUSSION}

\section{Payang Bugis}

The boat used to capture small pelagic fish around the rumpon was the small one. The term of payang Bugis become popular because fishermen firstly used capture device payang in Pasauran are from Bugis/ Celebes (around 1960), therefore it is called payang Bugis by Pasauran fishermen.

The length of payang Bugis boat on research location was 8-10 m, width 2-2.2 m, and depth 0.3$0.5 \mathrm{~m}$ (around 3-3.5 GT) with the power $20 \mathrm{PK}$. Consturction of payang Bugis with length $65-68 \mathrm{~m}$. Size of net variously from $1-17 \mathrm{~cm}$, mesh size of cod end was $1 \mathrm{~cm}$. The specification of payang Bugis shown on Table 2.

\subsection{Leaves Morphology in Visual}

Leaves morphology in visual on the different attractors were conducted in laboratory and in the sea water. On laboratory scale, the endurance those attractors in aquarium to see leaves colour, water colour and fibres leaves. The result showed that coconut leaves more endurance than the other leaves, between nypa leaves and areca, the nypa was more endurance than pinang leaves (Table 3 ).

Attractor leaves in sea waters showed that coconut leaves relatively more endurance than areca leaves and nypa leaves (Table 4). The condition of rumpon attractors installed in a waters in different season (MP1, MT, MP2, MB) showed that the resistence of attractors installed in the waters from the longest to the lowest was coconut leaves, nipah leaves and then pinang leaves. Rumpon with pinang leaves attractor only resisted in 15 days and became transparent and thin as well, nipah leaves became dark brown in 1921 days, whereas the coconut leaves became dark brown in 24-28 days. 
Table 2. Spesification of payang Bugis

\begin{tabular}{|c|c|c|c|c|c|c|c|}
\hline \multirow[b]{2}{*}{ Net Parts } & \multirow[b]{2}{*}{ Materials } & \multirow[b]{2}{*}{ Twine } & \multirow[b]{2}{*}{$\begin{array}{l}\text { Mesh size } \\
\text { (cm) }\end{array}$} & \multicolumn{2}{|c|}{ Length } & \multicolumn{2}{|c|}{ Width } \\
\hline & & & & $\begin{array}{l}\text { Act } \\
\text { (m) }\end{array}$ & $\begin{array}{l}\text { Jlh } \\
(\#)\end{array}$ & $\begin{array}{l}\text { Act } \\
\text { (m) }\end{array}$ & $\begin{array}{l}\text { Jlh } \\
(\#)\end{array}$ \\
\hline $\begin{array}{l}\text { Bunt } \\
\text { Net }\end{array}$ & $\mathrm{PE}$ & $210 \mathrm{D} \times 6$ & 1 & 2 & 125 & 25.6 & 1600 \\
\hline 1 & PE & $210 \mathrm{D} \times 3$ & 1 & 2 & 125 & 32 & 2000 \\
\hline 2 & PE & $210 \mathrm{D} \times 3$ & 1 & 2 & 125 & 32 & 2000 \\
\hline 3 & PE & $210 \mathrm{D} \times 3$ & 1 & 2 & 125 & 32 & 2000 \\
\hline 4 & PE & $210 \mathrm{D} \times 3$ & 1 & 2 & 125 & 32 & 2000 \\
\hline 5 & PE & $210 \mathrm{D} \times 3$ & 1 & 2 & 125 & 32 & 2000 \\
\hline Kerep Net & PE & $210 \mathrm{D} \times 9$ & 7 & 1 & 9 & 166.6 & 1500 \\
\hline Arang Net & PE & $210 \mathrm{D} \times 10$ & 10 & 1 & 9 & 83.3 & 750 \\
\hline $\begin{array}{l}\text { Sakam Net } \\
\text { Wing }\end{array}$ & PE & $210 \mathrm{D} \times 9$ & 25 & 0.5 & 9 & 8.6 & 155 \\
\hline 1 & PE & $210 \mathrm{D} \times 9$ & 17 & 48 & 900 & 8.3 & 155 \\
\hline 2 & PE & $210 \mathrm{D} \times 9$ & 17 & 48 & 900 & 8.3 & 155 \\
\hline Selvage 1 & PE & $210 \mathrm{D} \times 9$ & 17 & 1 & 5 & 3 & 15 \\
\hline Selvage 2 & PE & $210 \mathrm{D} \times 9$ & 17 & 1 & 5 & 3 & 15 \\
\hline Rope Parts & & Materials & $\begin{array}{l}\text { Diameter } \\
(\mathbf{m m})\end{array}$ & Le & & To & \\
\hline Selambar line & & PE & 10 & & & 2 & \\
\hline Outer line & & PE & 8 & & & 2 & \\
\hline Upper line & & PE & 8 & & & 2 & \\
\hline
\end{tabular}

Note $: \mathrm{PE}=$ Polyethylene, $\mathrm{Act}=$ actual, $\mathrm{Jlh}=$ number of mesh size

Table 3. Physical change of 3 natural attractor materials in the laboratory experiment

\begin{tabular}{|c|c|c|c|c|c|c|}
\hline \multirow{2}{*}{ Type of leaves } & \multirow{2}{*}{ Indicators } & \multicolumn{5}{|c|}{ Week- } \\
\hline & & 1 & 2 & 3 & 4 & 5 \\
\hline Nypa fructican & $\begin{array}{l}\text { Leaves' Color } \\
\text { Water's color } \\
\text { Microalgae on the leaves } \\
\text { Fibres on leaves }\end{array}$ & $\begin{array}{l}\text { Light green } \\
\text { clear } \\
\text { none } \\
\text { Pure }\end{array}$ & $\begin{array}{l}\text { dark green } \\
\text { clear } \\
\text { none } \\
\text { pure }\end{array}$ & $\begin{array}{c}\text { Brownish green } \\
\text { clear } \\
\text { less } \\
\text { blur }\end{array}$ & $\begin{array}{l}\text { dark brown } \\
\text { clear } \\
\text { much } \\
\text { blur }\end{array}$ & $\begin{array}{l}\text { Rotten } \\
\text { Clear } \\
\text { Much } \\
\text { blur }\end{array}$ \\
\hline Areca catechu & $\begin{array}{l}\text { Leaves' color } \\
\text { Water's color } \\
\text { Microalgae on leaves } \\
\text { Fibres on leaves }\end{array}$ & $\begin{array}{l}\text { Light green } \\
\text { Clear } \\
\text { None } \\
\text { Pure }\end{array}$ & $\begin{array}{l}\text { Dark green } \\
\text { Clear } \\
\text { None } \\
\text { Clear }\end{array}$ & $\begin{array}{c}\text { Brownish green } \\
\text { Clear } \\
\text { Less } \\
\text { Blur }\end{array}$ & $\begin{array}{l}\text { Transparant } \\
\text { Clear } \\
\text { Much } \\
\text { Vanished }\end{array}$ & $\begin{array}{l}\text { Rotten } \\
\text { Clear } \\
\text { Much } \\
\text { Vanished }\end{array}$ \\
\hline Coconut & $\begin{array}{l}\text { Leaves' color } \\
\text { Water's color } \\
\text { Microalgae on leaves }\end{array}$ & $\begin{array}{l}\text { Light green } \\
\text { Clear } \\
\text { None }\end{array}$ & $\begin{array}{l}\text { Dark green } \\
\text { Clear } \\
\text { Less }\end{array}$ & $\begin{array}{l}\text { Brownish green } \\
\text { Light brown } \\
\text { Less }\end{array}$ & $\begin{array}{l}\text { Dark brown } \\
\text { Brown } \\
\text { Less }\end{array}$ & $\begin{array}{l}\text { rotten } \\
\text { brown } \\
\text { Much }\end{array}$ \\
\hline & Fibres on leaves & Pure & Pure & Clear & Blur & Vanished \\
\hline
\end{tabular}


Table 4. Change of 3 natural attractor materials in the field/sea water

\begin{tabular}{|c|c|c|c|c|c|}
\hline \multirow{2}{*}{$\begin{array}{l}\text { Type of } \\
\text { leaves }\end{array}$} & \multirow{2}{*}{ Indicators } & \multicolumn{4}{|c|}{ Week } \\
\hline & & 1 & 2 & 3 & 4 \\
\hline \multirow[t]{3}{*}{ Nypa fructican } & Leaves' color & Light green & Dark brown & Rotten & \\
\hline & Alga and Avert. On leaves & None & Much & Much & \\
\hline & Fibres on leaves & Pure & Clear & Vanished & \\
\hline \multirow[t]{3}{*}{ Areca catechu } & Leaves' color & Light green & $\begin{array}{l}\text { Transparant } \\
\text { or rotten }\end{array}$ & & \\
\hline & Alga dan Avert. pada daun & None & Less & & \\
\hline & Fibres on leaves & Pure & Blur & & \\
\hline \multirow[t]{3}{*}{ Coconut } & Leaves' color & Light green & $\begin{array}{l}\text { Brownish } \\
\text { green }\end{array}$ & Dark brown & Rotten \\
\hline & Alga and Avert. On leaves & None & Less & Much & Much \\
\hline & Fibres on leaves & Pure & Pure & Clear & Blur \\
\hline
\end{tabular}

\subsection{Capture Result Composition}

Experimental fishing with payang around rumpon has captured 17 fish species and molluca (Table 5). Ten species are pelagic fish, i.e. green mackerel (Atule mate), yellowstripe mackerel (Selaroides leptolepis), striped mackerel (Rastrelliger kanagurta), bentong mackerel (Selar crumenophthalmus), scad mackerel (Decapterus russelli), layang scad (Decapterus macrosoma), frigate mackerel (Auxis thazard), fringescale sardine (Amblygaster sirm), rainbow runner (Elagatis bipinnulatus) and hardtail scad (Megalaspis cordyla). The others are demersal fish such as trevally (Caranx sexfasciatus), rabitfish (Siganus guttatus), eteman (Mene maculatus), bombay duck (Harpodon nehereus), Pomadasys macullatus, Abalistes and Indonesian oil sardine (Sardinella fimbriata). Molluca captured was squit (Loligo sp.). Composition of capture result and relative frequency for ten pelagic species each attractor per season can be seen on Table 6 and Picture 3.

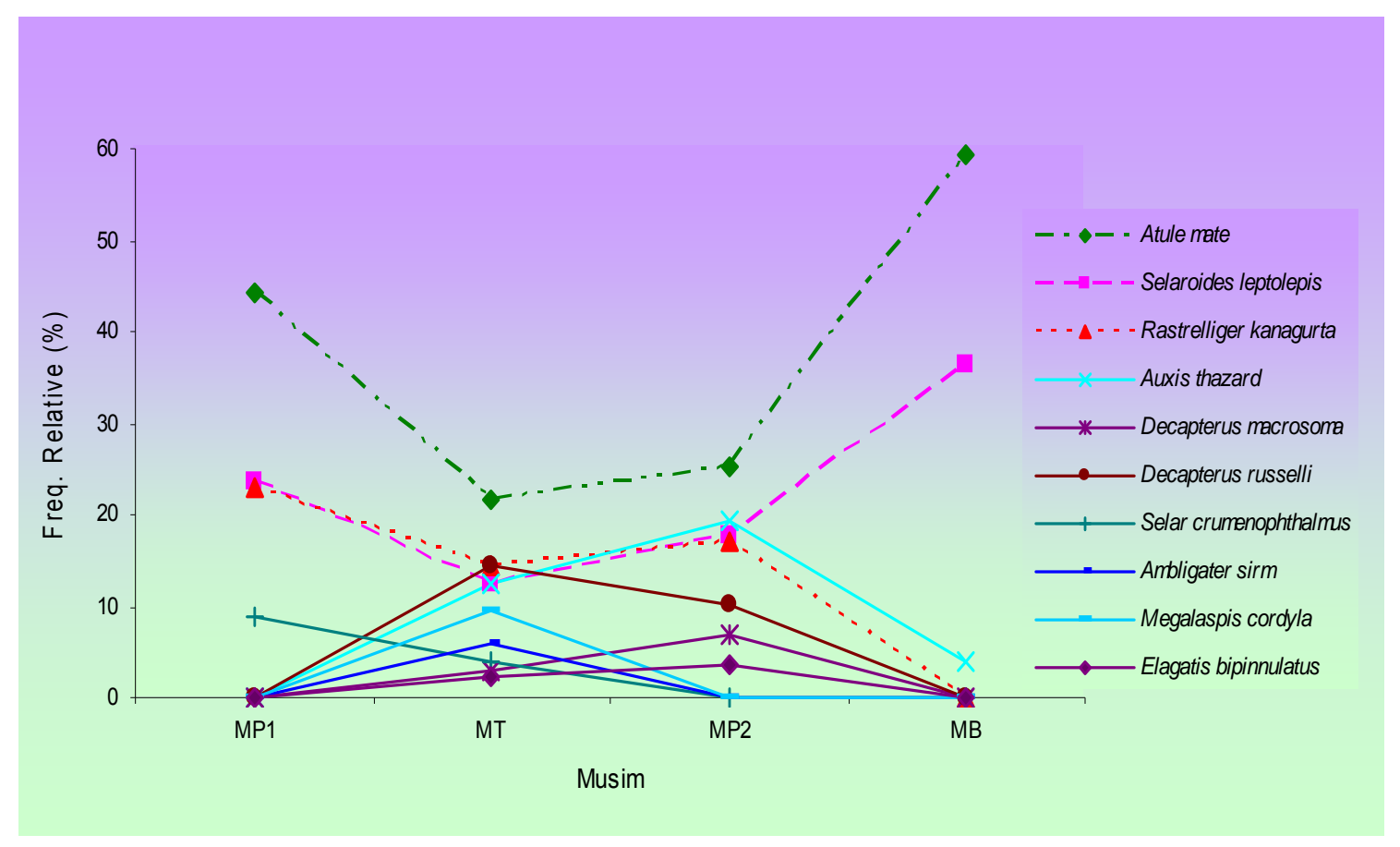

Picture 3. Relative frequency of capture result on the attractor materials in each season. 

in Pasuruan Waters, Province of Banten (Yusfiandayani R.)

Table 5. Existence fish species on capture result of payang around three rumpon with different materials

\begin{tabular}{|c|c|c|c|c|c|c|c|c|c|c|c|}
\hline \multirow{2}{*}{ No } & \multirow{2}{*}{ Fish Species } & \multirow{2}{*}{ Seasons } & \multicolumn{3}{|c|}{ Attractor Materials } & \multirow[b]{2}{*}{ No } & \multirow{2}{*}{ Fish Species } & \multirow{2}{*}{ Seasons } & \multicolumn{3}{|c|}{ Attractor Materials } \\
\hline & & & Nipa & Areca & Coconut & & & & Nipa & Areca & Coconut \\
\hline \multirow[t]{4}{*}{1} & Green mackerel & MP1 & $\sqrt{ }$ & $\sqrt{ }$ & $\sqrt{ }$ & 10 & Trevally & MP1 & - & - & - \\
\hline & (Atule mate) & MT & $\sqrt{ }$ & $\sqrt{ }$ & $\sqrt{ }$ & & (Caranx sexfasciatus) & MT & $\checkmark$ & $\sqrt{ }$ & $\sqrt{ }$ \\
\hline & & MP2 & $\sqrt{ }$ & $\sqrt{ }$ & $\checkmark$ & & & MP2 & - & - & - \\
\hline & & MB & $\sqrt{ }$ & $\sqrt{ }$ & $\checkmark$ & & & MB & - & - & - \\
\hline \multirow[t]{4}{*}{2} & Yellowstripe mackerel & MP1 & $\sqrt{ }$ & $\checkmark$ & $\checkmark$ & 11 & Eteman & MP1 & - & - & - \\
\hline & (Selaroides leptolepis) & MT & $\sqrt{ }$ & $\sqrt{ }$ & $\checkmark$ & & (Mene maculates) & MT & $\sqrt{ }$ & $\checkmark$ & $\sqrt{ }$ \\
\hline & & MP2 & $\sqrt{ }$ & $\sqrt{ }$ & $\sqrt{ }$ & & & MP2 & - & - & - \\
\hline & & MB & $\sqrt{ }$ & $\sqrt{ }$ & $\sqrt{ }$ & & & MB & - & - & - \\
\hline \multirow[t]{4}{*}{3} & Striped mackerel & MP1 & $\sqrt{ }$ & $\sqrt{ }$ & $\sqrt{ }$ & 12 & Rabitfish & MP1 & - & - & - \\
\hline & (Rastrelliger kanagurta) & MT & $\sqrt{ }$ & $\sqrt{ }$ & $\sqrt{ }$ & & (Siganus guttatus) & MT & $\sqrt{ }$ & $\sqrt{ }$ & $\sqrt{ }$ \\
\hline & & MP2 & $\sqrt{ }$ & $\sqrt{ }$ & $\sqrt{ }$ & & & MP2 & - & - & - \\
\hline & & MB & - & - & - & & & MB & - & - & - \\
\hline \multirow[t]{4}{*}{4} & Scad mackerel & MP1 & - & - & - & 13 & Rainbow runner & MP1 & - & - & - \\
\hline & (Decapterus russelli) & MT & $\checkmark$ & $\sqrt{ }$ & $\sqrt{ }$ & & (Elegatis bipinnulatus) & MT & - & - & $\sqrt{ }$ \\
\hline & & MP2 & $\sqrt{ }$ & $\checkmark$ & $\sqrt{ }$ & & & MP2 & - & - & $\sqrt{ }$ \\
\hline & & MB & - & - & - & & & MB & - & - & - \\
\hline \multirow[t]{4}{*}{5} & Layang scad & MP1 & - & - & - & 14 & Hardtail scad & MP1 & - & - & $\sqrt{ }$ \\
\hline & (Decapterus macrosoma) & MT & - & - & $\sqrt{ }$ & & (Megalaspis cordyla) & MT & - & - & $\sqrt{ }$ \\
\hline & & MP2 & $\checkmark$ & $\sqrt{ }$ & $\sqrt{ }$ & & & $\mathrm{MP} 2$ & - & - & - \\
\hline & & MB & - & - & - & & & MB & - & - & - \\
\hline \multirow[t]{4}{*}{6} & Frigate mackerel & MP1 & - & - & - & 15 & Bombay duck & MP1 & $\checkmark$ & - & - \\
\hline & (Auxis thazard) & MT & $\sqrt{ }$ & $\sqrt{ }$ & $\sqrt{ }$ & & (Harpodon nehereus) & MT & - & - & $\sqrt{ }$ \\
\hline & & MP2 & $\sqrt{ }$ & $\sqrt{ }$ & $\sqrt{ }$ & & & MP2 & - & - & - \\
\hline & & MB & - & - & $\sqrt{ }$ & & & MB & - & - & - \\
\hline \multirow[t]{4}{*}{7} & Bentong mackerel & MP1 & $\sqrt{ }$ & $\sqrt{ }$ & $\sqrt{ }$ & 16 & Pomadasys macullatus & MP1 & $\sqrt{ }$ & - & - \\
\hline & (Selar crumenophthalmus) & MT & $\sqrt{ }$ & - & $y$ & & & MT & - & - & $\checkmark$ \\
\hline & & MP2 & - & - & - & & & MP2 & - & - & - \\
\hline & & MB & - & - & - & & & $\mathrm{MB}$ & - & - & - \\
\hline \multirow[t]{4}{*}{8} & Fring escale sardine & MP1 & - & - & - & 17 & Abalistes & MP1 & $\checkmark$ & - & $\sqrt{ }$ \\
\hline & (Amblygaster sirm) & MT & $\checkmark$ & $\sqrt{ }$ & $\sqrt{ }$ & & & MT & - & - & - \\
\hline & & MP2 & - & - & - & & & MP2 & - & - & - \\
\hline & & MB & - & - & - & & & MB & - & - & - \\
\hline \multirow[t]{4}{*}{9} & Indonesian oil sardine & MP1 & - & - & - & 18 & Squid (Loligo sp.) & MP1 & - & $\sqrt{ }$ & - \\
\hline & (Sardinella fimbriata) & MT & $\sqrt{ }$ & $\sqrt{ }$ & $\sqrt{ }$ & & & MT & - & - & $\sqrt{ }$ \\
\hline & & MP2 & - & - & - & & & MP2 & - & - & - \\
\hline & & MB & - & - & - & & & MB & - & - & - \\
\hline
\end{tabular}

Note $: \sqrt{ }$ indicates fish available on time and those attractors

Table 6

Composition of capture result pelagic fish

\begin{tabular}{lccccc}
\hline \multirow{2}{*}{ Attractors } & \multicolumn{4}{c}{ Capture fish/season } & \multirow{2}{*}{ Total } \\
\cline { 2 - 5 } & MP1 & MT & MP2 & MB & \\
\hline Nypa fructican & 122 & 166 & 78 & 24 & 390 \\
Areca catechu & 25 & 57 & 27 & 16 & 125 \\
Coconut & 165 & 358 & 140 & 34 & 674 \\
Total & 312 & 581 & 245 & 74 & 1212 \\
\hline
\end{tabular}

Based on test analyses Kruskal-Wallis, it shows that capture result for the total result of payang is significantly different $(P: 0.0 ; P<0.05)$ based on materials and seasons. From Duncan Signed Rank
Experiment, it is derived that the average of capture result payang for total capture on rumpon with coconut leaves in east season was the highest (Table 7). Based on type of rumpon material, it can be seen that the average of capture result on coconut leaves was not different significantly among the east season, season 1 and 2, but those are significantly higher than on the west season. The similar pattern also can be seen on rumpon with nipa leaves where capture result on season 1 and east season was significantly higher than on west season, whereas there was no significantly difference with the other seasons. If it is compared to the other leaves, areca leaves give more homogenic respons with low capture result and do not show significant difference on seasons. 
Table 7.

Result Analyses on Duncan Signed Rank Experiment for Dominant Fish

\begin{tabular}{|c|c|c|c|c|c|c|c|}
\hline \multirow{2}{*}{ Treatment } & \multicolumn{6}{|c|}{ Fish Species } & \multirow{2}{*}{ Total } \\
\hline & AT & SL & AM & RK & SF & DR & \\
\hline 1 & $3.33^{b c}$ & $3.11^{\mathrm{bc}}$ & $6.11^{\text {cde }}$ & $0.00^{\mathrm{a}}$ & $3.33^{\mathrm{bcd}}$ & $0.00^{a}$ & $15.89^{b c}$ \\
\hline 2 & $0.56^{a}$ & $0.33^{a}$ & $1.44^{\mathrm{bc}}$ & $0.00^{\mathrm{a}}$ & $1.11^{\mathrm{a}}$ & $0.00^{a}$ & $3.44^{\mathrm{a}}$ \\
\hline 3 & $4.11^{\mathrm{C}}$ & $4.78^{\mathrm{cd}}$ & $7.78^{\text {de }}$ & $0.00^{a}$ & $4.11^{c d}$ & $0.00^{a}$ & $20.78^{c}$ \\
\hline 4 & $2.56^{\mathrm{bc}}$ & $2.56^{\mathrm{bc}}$ & $4.00^{\mathrm{bcd}}$ & $2.78^{c}$ & $2.33^{a b c}$ & $3.67^{c}$ & $17.89^{\mathrm{bc}}$ \\
\hline 5 & $0.56^{a}$ & $0.33^{a}$ & $1.78^{a b}$ & $1.00^{a b}$ & $1.33^{a b}$ & $1.11^{\mathrm{ab}}$ & $6.11^{a}$ \\
\hline 6 & $5.00^{c}$ & $5.22^{d}$ & $8.22^{e}$ & $5.44^{d}$ & $5.67^{d}$ & $6.33^{d}$ & $35.89^{d}$ \\
\hline 7 & $1.56^{\mathrm{ab}}$ & $1.56^{\mathrm{ab}}$ & $2.22^{\mathrm{ab}}$ & $1.56^{\mathrm{bc}}$ & $0.44^{\mathrm{a}}$ & $1.78^{\mathrm{bc}}$ & $9.11^{\mathrm{ab}}$ \\
\hline 8 & $0.56^{\mathrm{a}}$ & $0.33^{a}$ & $1.00^{a}$ & $0.56^{\mathrm{ab}}$ & $0.44^{\mathrm{a}}$ & $0.56^{a}$ & $3.44^{\mathrm{a}}$ \\
\hline 9 & $3.11^{b c}$ & $2.89^{b c}$ & $3.67^{\mathrm{abcd}}$ & $2.56^{\mathrm{c}}$ & $1.89^{a b c}$ & $2.33^{c}$ & $16.44^{\mathrm{bc}}$ \\
\hline 10 & $0.00^{\mathrm{a}}$ & $1.00^{\mathrm{ab}}$ & $3.00^{\mathrm{abc}}$ & $0.00^{\mathrm{a}}$ & $1.17^{\mathrm{ab}}$ & $0.00^{a}$ & $5.17^{\mathrm{a}}$ \\
\hline 11 & $0.00^{a}$ & $1.00^{\mathrm{ab}}$ & $1.67^{\mathrm{ab}}$ & $0.00^{a}$ & $0.33^{a}$ & $0.00^{a}$ & $3.00^{a}$ \\
\hline 12 & $0.50^{\mathrm{a}}$ & $2.50^{\mathrm{bc}}$ & $2.67^{\mathrm{ab}}$ & $0.00^{a}$ & $2.17^{\mathrm{abc}}$ & $0.00^{a}$ & $7.83^{\mathrm{ab}}$ \\
\hline
\end{tabular}

Note : ab, bc, cd, abc, bcd, cde, abcd on the same column indicate significant different results

$\mathrm{AT}=$ Auxis thazard, $\mathrm{SL}=$ Selaroides leptolepis, $\mathrm{AM}=$ Atule mate, $\mathrm{RK}=$ Rastrelliger kanagurta

$\mathrm{DR}=$ Decapterus russelli

1 Captured fish around nypa fructican leaves in MP1

2 Captured fish around areca catechu leaves in MP1

3 Captured fish around coconut leaves in MP1

4 Captured fish around nypa fructican leaves in MT

5 Captured fish around areca catechu leaves in MT

6 Captured fish around coconut leaves in MT

7 Captured fish around nypa fructican leaves in MP2

8 Captured fish around areca catechu leaves in MP2

9 Captured fish around coconut leaves in MP2

10 Captured fish around nypa fructican leaves in MB

11 Captured fish around areca catechu leaves in MB

12 Captured fish around coconut leaves in MB

Pelagic fish captured were green mackerel (Atule mate), yellowstripe mackerel (Selaroides leptolepis), striped mackerel (Rastrelliger kanagurta), frigate mackerel (Auxis thazard), layang scad (Decapterus macrosoma), bentong mackerel (Selar crumenopthalmus), fringescale sardine (Amblygaster sirm), hardtail scad (Megalaspis cordyla) and rainbow runner (Elegatis bipinnulatus). Captured small pelagic fish around rumpon was normal because they are commonly available on the upper layer. Therefore, rumpon attractor materials are usually concentrated to be installed on the upper part.

In this research, the writer was also catch a lot of rabitfish on East season. Captured demersal fish that commonly associate with seaweed probably caused by rumpon installation more closely to dead reef (gosong).

Research conducted on season 1 in March, east season in June, season 2 in September and west season in December showed that fish captured by using payang Bugis are selar hijau (Atule mate) dan selar kuning (Selaroides leptolepis). It is based on fishery statistic data and Monintja's research (2003) which showed that fish used to capture along the year by payang Bugis and handline are green mackerel (Atule mate) dan yellowstripe mackerel (Selaroides leptolepis). Whereas, fish which stay temporarily are striped mackerel (Rastrelliger kanagurta), frigate mackerel (Auxis thazard), layang scad (Decapterus macrosoma), scad mackerel (Decapterus russelli) dan fringescale sardine (Amblygaster sirm). It is only based on characteristic of family carangidae which generally live in continental shelf waters, while some of scombridae fish are neritic and others are oceanic.

The smallest captured fish around rumpon called green mackerel (atule mate) were derived on season 1 with size 5.3 and weight $9.8 \mathrm{gr}$. The biggest size of captured fish around rumpon derived on east season are $28.0 \mathrm{~cm}$ and weight $356.5 \mathrm{gr}$ were frigate mackerel (auxis thazard). Generally, in each captured fish size was relatively small and short. Based on length at first maturity, those fish captured in immature condition. Captured fish on rumpon and in all season had gonado with GSI I, II, and III in series are $4 \%$, $33 \%$ and $35 \%$. Thus, it can be assured that associated and captured fish around the rumpon are immature 
fish that can endanger sustainability fish resource in Pasauran waters.

This result showed that $72.1 \%$ of fish were captured around rumpon with GSI I-III or less than length at first maturity. In the other hand, the amount of fish with GSI I-III were $24.8 \%$ and $3.1 \%$. Shallow sea rumpon utilization as one of the auxiliary gears in capturing fish was worried to give negative effect to sustainability of fish resource in Pasauran waters. It is open access and it should be revised in controlling fishery capture. It is also related with international issue since the International Conference about Fish Aggregating Device in Martinique, France in 1999. This issue was based on Code of Conduct for Responsible Fisheries released by FAO in 1995. The international issue was about FAD as auxiliary device to capture immature fish have dangered the sustainability fish resource in waters. It was because the capture device purse seine developing rapidly in Pasific Ocean on drifting fish aggregating device had caught small and immature tuna fish. There were some pro and controversy because FAD was auxiliary device assured very effective to capture fish by using purse seine device. Purse seine was an effective capture device around FAD, only trawl that can be used as comparison capture device in capture fish effectivity.

\subsection{Gonado Maturity Level/Gonado Somato Index}

\section{(1) Season 1}

312 individual fish consisted of 10 species which were captured around rumpon using payang Bugis, 5 species captured from individual that had gonado with GSI I. And three of ten species with GSI V had been captured, i.e. green and yellow mackerel, and striped mackerel (Rastrelliger kanagurta). Fish composition from GSI | $4.2 \%$, GSI II $26.6 \%$ and GSI III $39.4 \%$.

\section{(2) East Season}

581 individual fish consist of 10 species which were captured around rumpon using payang, only one species captured with no GSI I i.e. yellowstripe mackerel. There were not captured individual fish with GSI V from three of ten species: layang scad, bentong mackerel and rainbow runner. Fish composition from GSI $3.7 \%$, GSI II $37.2 \%$ and GSI III $32.6 \%$.

\section{(3) Season 2}

245 individual fish consist of ten species which were captured around rumpon using payang, three species were captured with no GSI I, i.e. bentong mackerel, fringescale sardine and rabitfish. There were captured individual fish with GSI V from four of ten species, they were green and yellowstripe mackerel, striped mackerel and frigate mackerel. Fish composition from GSI I 4.7\%, GSI II 35.2\% and GSI III $33.1 \%$.

\section{(4) West Season}

74 individual fish consist of ten species which were caputured around rumpon using payang, one species was captured with GSI I i.e. green mackerel. Individual fish with GSI V from two of ten species were captured i.e. green and yellowstriped mackerel. Fish composition from GSI I 1.4\%, GSI II $23.0 \%$ and GSI III $41.9 \%$.

Generally, captured fish with GSI I 4\%, GSI II 33\% and GSI III 35\% (Picture 4). Whereas, captures fish with GSI IV $25 \%$ and GSI V $3 \%$.

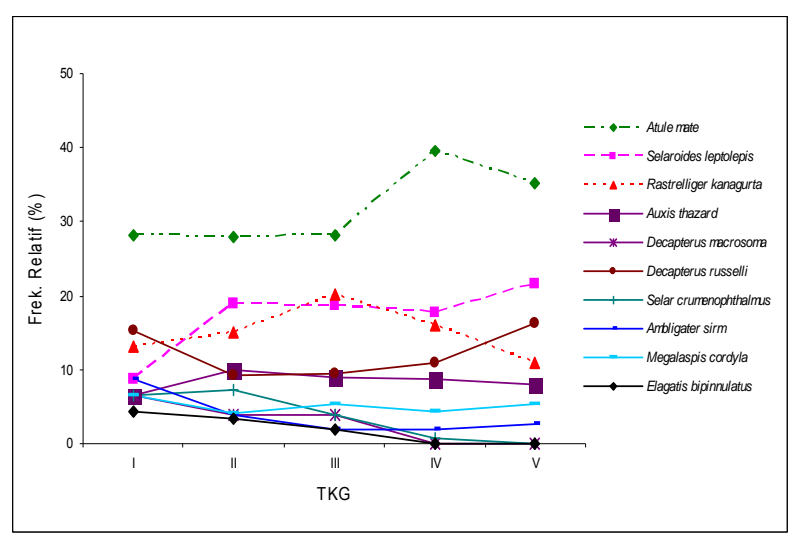

Picture 4. Frequency relative of Gonado Somato Index of Captured Fish

\section{CONSLUSION AND SUGGESTION}

\subsection{Conclusion}

(1) The great number and variety of fish species on different season obtained from fish capturing around rumpon whose attractors are from coconut leaves.

(2) The captured fish on rumpon have gonado with GSI I, II, III, periodically $4 \%, 33 \%$, and $35 \%$. Therefore, it can be assured that captured fish around rumpon are immature fish so that those fish have not done their reproduction yet. Fish capturing gonado with GSI I, II, and III may cause recruitment overfishing. 


\subsection{Suggestions}

(1) It needs further research on capture effort on shallow sea rumpon by using payang Bugis to know the optimum number of rumpon.

(2) It needs to apply closed and open season area in Pasauran waters adapted with determining of fish capture time to avoid capturing immature fish.

\section{REFERENCES}

A.P.H.A., 1989. Standard Methods for Examination of Water and Wastewater. 17 th Edition, Washington DC. P. : 1044-1075.

Barus, H. R., M. Linting, N. Naamin, S. Ilyas, M. Badrudin, C. Nasution, E. M. Amin, B. Gafa dan Sarjana. 1992. Pedoman Teknis Peningkatan Produksi dan Efisiensi melalui Penerapan Teknologi Rumpon. Departemen Pertanian Badan Penelitian dan Pengembangan Pertanian, Pusat Penelitian dan Pengembangan Perikanan. Jakarta. 87 hal.

Castro, J. J., Jose A. S., and A. T. S. Ortega. 2002. A general Theory on Fish Aggregation to Floating Objects : An Alternative to the Meeting Point Hypothesis. Fish Biology and Fisheries, 11 : 255277.

Cotel, P. and D. Petit. 1996. Target Strength Measurements on Three Pelagic Fishes from the Java Sea. Fourth Asian Fisheries Forum, 16-20 October 1995, Beijing. P. 109-117.

Dagorn, L., Kosse, E., Bach, P. and Bertrand, A. 2000. Modelling Tuna Behaviour near Floating Objects : from Individuals to Aggregations. Aquatic Living Resources. 13 : 203-211

Dinas Perikanan Dati II Kabupaten Serang. 1999. Monografi Perikanan Kabupaten Pandeglang (tidak dipublikasikan).

Direktorat Jenderal Perikanan. 1995. Penggunaan payaos/rumpon di Indonesia. Jakarta. $11 \mathrm{hal}$.

Effendie, M. I. 1979. Metode Biologi Perikanan. Yayasan Dewi Sri. Bogor. Hal. 15-85.

FAO. 1995. Code of Conduct for Responsible Fisheries. Rome, Italy. $41 \mathrm{p}$.

FAO. http://www.fishbase.org.
FAO. 1996. Pedoman Teknis untuk Perikanan Bertanggungjawab. Operasi Penangkapan Ikan. Diterjemahkan oleh Direktorat Jenderal Perikanan bersama Pusat Penelitian dan Pengembangan Perikanan, Badan Penelitian dan Pengembangan Pertanian dan Institut Pertanian Bogor, 1999, Fishing Operations. 115 hal.

Fischer, W. and P. J. P. Whitehead. 1974. FAO Species Identification Sheets for Fishery Purposes. Eastern Indian Ocean (Fishing Area 57) and Western Central Pasific (Fishing Area 71). Vol. Il dan III. FAO, Rome.

Freon, P. and L. Dagorn. 2000. Review of Fish Associate Behaviour : Toward a Generalisation of The Meeting Point Hypothesis. Fish Biology and Fisheries, 10 : 183-207.

Gooding, R. M. and J. J. Magnuson. 1967. Ecological Significance of A drifting Object to Pelagic Fishes. Pasific Science, 21 : 486-497.

Grofit, E. 1980. The Fishing Technology Unit (FTU). FAO Fisheries Technical Papers. FAO. Rome. 48 p.

MacLennan, D. N. and E. John Simmonds. 1992. Fisheries Acoustics. Chapman and Hall. LondonNew York-Tokyo-Melbourne-Madras. $325 \mathrm{p}$.

Mardiana, N. 1999. Prosedur Analisis Tanaman. Unit Penelitian Bioteknologi Tanaman. UPT Bioteknologi, Bogor. 24 hal.

Merta, I.G.S., S. Nurhakim dan J. Widodo. 1998. Sumberdaya perikanan pelagis kecil. dalam potensi dan penyebaran sumberdaya ikan laut di perairan Indonesia. Komisi Nasional Pengkajian Stok Sumberdaya Ikan Laut, Jakarta. Hal 89-106.

Mitson, R. B. 1983. Fisheries Sonar. Fishing News Books Ltd. Farnham, Surrey, England. 287 p.

Monintja, D. R. 1993. Study on the Development of Rumpon as Fish Aggregating Devices (FADs). Maritek, Bulletin ITK, FPIK-IPB. 3(2) : 137 p.

Monintja, D. R., J. Widodo dan F. A. Sondita. 2003. Pengkajian Terhadap Pemanfaatan Rumpon untuk Penangkapan Ikan Pelagis : Antisipasi Terhadap Implementasi Code of Conduct for Responsible Fisheries. Laporan Riset Unggulan Terpadu VIII. Kementrian Riset dan Teknologi Republik Indonesia, Lembaga IImu Pengetahuan Indonesia, Jakarta. 96 hal. (Tidak dipublikasikan). 
Nasoetion, A. H., dan Barizi. 1980. Metode Statistika untuk Menarik Kesimpulan. PT. Gramedia. Jakarta. 223 hal.

Steel, R.G.D. and Torrie, J.H., 1989. Prinsip dan Prosedure Statistika (suatu pendekatan biometrik). Alih bahasa B. Sumantry. P.T. Gramedia, Jakarta. 748 p.

Taquet, M., L. Reynal and M. Laurans. 1999. Do FADs Influence The Geographical Distribution of Dolphinfish (Coryphaena hippurus). Peche Thoniere et Dispositifs de Concentration de Poissons. Caraibe-Martinique, 15-19 October 1999. p. 528-536.

Tim Pengkajian Rumpon Fakultas Perikanan Institut Pertanian Bogor. 1987. Laporan Akhir Survai Lokasi dan Desain Rumpon di Perairan Ternate, Tidore, Bacan dan sekitarnya. Laporan. Jurusan
Pemanfaatan Sumberdaya Perikanan. Fakultas Perikanan. Institut Pertanian Bogor, Bogor. Hal. V. 54-58 (Tidak dipublikasikan).

Widodo, J. 1997. Review of The Small Pelagic Fisheries in Indonesia. Proceedings Asia Pasific Fisheries Commission Food and Agriculture Organization of The United Nations Office for Asia and the Pasific. Bangkok, Thailand. P. 199-226.

Widodo, J., K. A. Aziz, B. E. Priyono, G. H. Tampubolon, N. Naamin dan A. Djamali (Editor). 1998. Potensi dan Penyebaran Sumber Daya Ikan Laut di Perairan Indonesia. Komisi Nasional Pengkajian Stok Sumber Daya lkan Laut. Jakarta. 42 hal.

Yamaji, L. 1976. Illustration of Marine Plankton of Japan. Hoikusha Publishing Co. Ltd, Japan. 360 p. 